\title{
CATÁLOGO POLÍNICO DO PARQUE ESTADUAL DE VILA VELHA, PARANÁ - 2ª. PARTE
}

\author{
POLLEN CATALOGUE \\ OF VILA VELHA STATE PARK - $2^{\text {st }}$ PART
}

\author{
INÊS JANETE MATTOZO TAKEDA ${ }^{1}$ \\ PAULO VITOR FARAGO ${ }^{2}$ \\ MELISSA KOCH FERNANDES DE SOUZA ${ }^{3}$ \\ VANESSA VIEIRA GELINSKI ${ }^{2}$
}

1 Professora da UEPG e da UNICENP

2 Farmacêutico (UEPG)

3 Biólogo (UEPG)

\begin{abstract}
RESUMO
A palinologia apresenta várias aplicações. Em muitos casos, o único elemento de identificação é o grão de pólen, obtido de uma planta previamente determinada e referendada em um herbário. Foram analisadas sete espécies pertencentes a sete famílias, obtidas nos acervos dos herbários UEPG e UPCB, seguindo um planejamento para a abordagem de diferentes famílias, possibilitando trabalhos futuros relacionados às aplicações deste catálogo. Os grãos foram submetidos à acetólise. Para a montagem das lâminas foi utilizada a gelatina-glicerinada de Kisser, seguindo-se a lutagem com parafina. A caracterização foi efetuada através de vários critérios, como: forma, tamanho e ornamentação da exina. Os táxons estudados foram: Asclepiadaceae - Ditassa edmundoi Fontella et Valente, Asteraceae - Vernonia nudiflora Less, Begoniaceae - Begonia fruticosa (Kl.) A. DC., Berberidaceae Berberis laurina Billb., Bignoniaceae - Jacaranda oxyphylla Cham., Caryophyllaceae - Cerastium dicrotrichum Fengl., Commelinaceae - Tradescantia crassula Link et Otto. A família Asteraceae é euripalinológica e apresenta vários tipos polínicos.
\end{abstract}


Palavras-chave: catálogo polínico; Parque Estadual de Vila Velha; Região dos Campos Gerais

\section{Introdução}

A análise da morfologia polínica tem importância para diferentes ciências: Melissopalinologia, Farmacognosia, Paleobotânica, Paleoecologia, Bromatologia, Farmacologia, Fitoecologia, Zooecologia, Sedimentologia, Medicina Legal, Taxonomia Vegetal, Toxicologia, entre outras.

Na maioria das aplicações da palinologia, o único elemento de identificação é o grão de pólen. Pelas diferentes características que podem ser observadas nessa estrutura, é possível a determinação da planta em diversos níveis taxonômicos, sendo algumas vezes considerada a família, o gênero ou o tipo (que envolvem vários gêneros) e, mais raramente, a espécie.

Este estudo consiste no início de uma catalogação polínica e na confecção de um laminário palinológico de referência, visando estudos nas ciências indicadas acima.

\section{Material e métodos}

A Região dos Campos Gerais situa-se no Segundo Planalto Paranaense guardando em sua paisagem natural um ambiente onde predominam campos e cerrados. A vegetação florestal que ocorre na área é em forma de capões e matas de galeria, dominada pela Araucaria angustifolia (Bertol.) Kuntze.

Foram selecionadas sete espécies pertencentes a sete famílias. As espécies foram selecionadas de acordo com o material disponível para análise de grão de pólen. O material polínico foi obtido de flores de exsicatas do acervo dos Herbários UEPG (Universidade Estadual de Ponta Grossa) e UPCB (Universidade Federal do Paraná) e foi submetido à acetólise, de acordo com o método de Erdtman (1952, 1969), modificado por SalgadoLaboriau (1973). Este método permite a comparação de pólens atuais com material fossilizado. Para a montagem das lâminas foi usada gelatinaglicerinada de Kisser (SALGADO-LABOURIAU, 1973) e em seguida, foi feita a lutagem com parafina. 
A caracterização dos grãos de pólen foi efetuada através dos seguintes critérios: relação P/E (ERDTMAN, 1952), determinação da área polar, forma, dimensões e localização de aberturas e ornamentação da exina. Fundamentou-se as descrições dos pólens em Miranda e Andrade (1990) e Markgraf e D’Antoni (1978).

Para as medições foram utilizados os critérios de Salgado-Labouriau (1966, 1973). A terminologia empregada foi a de Erdtman $(1952,1969)$.

Os grãos de pólen foram ilustrados por fotografias que foram tomadas em fotomicroscópio Zeiss MC-80.

O tratamento estatístico das grandezas medidas em 10 grãos de pólen pertencente ao mesmo táxon, permitiu o cálculo da média aritmética e do desvio padrão (tabela 1).

\section{Resultados e discussão}

\section{ASCLEPIADACEAE}

NOME CIENTÍFICO: Ditassa edmundoi Fontella et Valente.

NOME VULGAR: Leiteira-brava

REFERÊNCIA: UPCB 7751.

ECOLOGIA: Planta escandente em floresta com araucária.

PÓLEN: em políade ovalada, com número de tétrades igual a oito. Medindo, em média, 59,9 mm por 26,6 mm. As tétrades são imperfeitamente tetraédricas. A exina é espessa, sendo a sexina mais espessa que a nexina. A sexina é mais espessa na parte externa dos grãos que formam a políade. O pólen apresenta-se psilado, sem báculos. Observação: não foi possível observar as aberturas (prancha 1: 1A).

No Parque Estadual de Vila Velha ocorrem três gêneros da família Asclepiadaceae: Ditassa, Nautonia, Oxypetalum, sendo os dois primeiros gêneros representados cada um por uma única espécie e o último, o mais representado, com seis espécies (HATSCHBACH e MOREIRA FILHO, 1972).

Nesta família ocorrem grãos de pólen unidos em tétrades (gêneros: Cryptolepis, Curroria, Periploca, Tacazzea) (ERDTMAN, 1952) ou em políneas (gênero: Tweedia) (MARKGRAF e D’ANTONI, 1978). A espécie Ditassa edmundoi é considerada rara e endêmica por Cervi e Hatschbach (1990). 
ASTERACEAE

NOME CIENTÍFICO: Vernonia nudiflora Less.

NOME VULGAR: Alecrim-do-campo.

REFERÊNCIA: UPCB 6307.

ECOLOGIA: ocorre principalmente em solos secos e pedregosos nos campos e beira de mata.

PÓLEN: tricolporado, subprolato (subesferoidal), com P/E =1,19624. Tamanho médio. A exina possui báculos bem evidentes. A sexina é mais espessa que a nexina. As paredes da sexina são muito espessas. O pólen apresenta-se equinado, lofado. (prancha 1: 2A, 2B, 2C e 2D).

A família Asteraceae é uma das mais representadas no Parque Estadual de Vila Velha com 91 espécies, em 32 gêneros, de acordo com Hatschbach e Moreira Filho (1972) e levantamentos efetuados no herbário UPCB. O gênero Vernonia é o que apresenta maior número de espécies.

A caracterização de gêneros e/ou tribos para a família Asteraceae é perfeitamente possível, graças à grande variedade de forma, tamanho e estratificação da exina de seus grãos. Para tanto, faz-se necessária a complementação do estudo polínico através de microscopia eletrônica (TOMB et al., 1974; REIS, 1979).

Stix (1960), analisando a morfologia dos grãos de pólen em microscopia de luz ultravioleta, considerou 45 tipos para Asteraceae, de acordo com linhas de parentesco, onde o tipo polínico pode abranger várias tribos, subtribos, um gênero ou mesmo uma espécie.

Cervi e Hatschbach (1990) consideram Gochnatia orbicularis (Malme) Cabr., G. argysrea Malme, Baccharis aphylla (Vell.) DC., Isostigma speciosum Less., Pamphalea smithii Cabr., Chaptalia graminifolia Dusén, como espécies endêmicas e raras. Constando na lista vermelha das espécies em extinção no estado do Paraná, a espécie G. argysre Malme: em perigo, I. speciosum Less.: rara, Pamphalea smithii Cabr.: em perigo, Trichocline linearifolia Malme: em perigo, Vernonia sessilifolia Less.: em perigo.

Considerando os pontos acima relatados, é necessário o aumento da amostragem para a família Asteraceae, uma vez que a espécie estudada é representativa somente para a tribo Vernoniae.

BEGONIACEAE

NOME CIENTÍFICO: Begonia fruticosa (Kl.) A. DC.

NOME VULGAR: Begônia-arbustiva, azeda, coração-de-estudante. REFERÊNCIA: HUEPG 5453.

ECOLOGIA: Espécie seletiva higrófita e ciófita (umbrófila), é ge- 
ralmente mais freqüente nas depressões, fundo dos vales e grutas, bem como em solos pedregosos, onde o microclima do ambiente está continuamente quase saturado pela umidade.

PÓLEN: tricolporado, circulaperturado, prolato, com P/E = 1,5. Tamanho muito pequeno. Deduz-se que a sexina é mais espessa que a nexina pois a nexina é muito delgada. O pólen apresenta-se irregularmente reticulado (prancha 2: 3A, 3B e 3C).

De acordo com Erdtman (1952), os grãos de pólen da família Begoniaceae apresentam-se tricolporados, de prolato a perprolatos, pequenos. Hatschbach e Moreira Filho (1972) e Cervi e Hatschbach (1990) citam apenas a espécie Begonia setosa Kl. para o local de estudo.

Pela pequena representação da família na unidade de conservação, pela semelhança na morfologia polínica das espécies de Begonia e pelo pequeno tamanho do grão de pólen, considera-se que $B$. fruticosa pode representar a espécie ocorrente no Parque Estadual de Vila Velha.

BERBERIDACEAE

NOME CIENTÍFICO: Berberis laurina Billb.

NOME VULGAR: São João, Espina amarilla (Uruguai).

REFERÊNCIA: UPCB 2269.

ECOLOGIA: Arbusto característico e exclusivo da zona dos pinhais, com ampla e expressiva dispersão pelo planalto.

PÓLEN: fossaperturado, com aberturas irregulares, prolato-esferoidal (subesferoidal), com $\mathrm{P} / \mathrm{E}=1,01053$. Tamanho médio. A exina é relativamente grossa e mais delgada na região dos colpos. A sexina é mais espessa que a nexina. O pólen apresenta-se reticulado (prancha 2: 4A).

Berberis laurina Bill. é a única espécie da família Berberidaceae que ocorre no Parque Estadual de Vila Velha (HATSCHBACH e MOREIRA FILHO, 1972). As principais características dessa espécie são comuns a outras espécies do mesmo gênero, como mostra Markgraf e D’ Antoni (1978) e Erdtman (1952).

BIGNONIACEAE

NOME CIENTÍFICO: Jacaranda oxyphylla Cham.

NOME VULGAR: Carobinha do campo.

REFERÊNCIA: UPCB 5533.

ECOLOGIA: Planta do campo, muito abundante e expressiva no campo seco, constituindo formações bastante densas nas depressões.

PÓLEN: tricolporado, imperfeitamente fossaperturado, prolato, com $\mathrm{P} / \mathrm{E}=1,45164$. Tamanho médio. A sexina é mais espessa que a nexina. Os 
colporos são longos, quase até o centro. O pólen apresenta-se tectado, sem ornamentação (prancha 2: 5A e 5B).

Segundo Hatschbach e Moreira Filho (1972) ocorrem 4 espécies de Bignoniaceae no Parque Estadual de Vila Velha, sendo 2 espécies de Jacaranda.

Erdtman (1969) relata a família Bignoniaceae como euripalinológica. De acordo com Markgraf e D’Antoni (1978), Jacaranda mimosifolia D. Don. apresenta características semelhantes à J. oxyphyla descrita neste estudo.

CARYOPHYLLACEAE

NOME CIENTÍFICO: Cerastium dicrotrichum Fengl.

NOME VULGAR: Cerastio.

REFERÊNCIA: UPCB 7206.

ECOLOGIA: Planta de campo seco.

PÓLEN: esferoidal, com P/E = 1. Tamanho médio. A exina é homogênea em toda a superfície do grão. A sexina é mais espessa que a nexina. O pólen apresenta-se homogeneamente reticulado, pantoporado e com os báculos espessos e paralelos (prancha 2: 6A e 6B).

De acordo com levantamentos nos herbário UEPG e UPCB, ocorrem 3 espécies no local de estudo: Cerastium dicrotrichum Fengl., Polycarpaea corymbosa (L.) Lam., Arenaria lanuginosa (Mx.) Rolvb. Como no próprio parque existe uma área para experimentos agrícolas, coordenados pelo IAPAR (Instituto Agronômico do Paraná), ocorrem também diversas espécies de Caryophylaceae que são invasoras de culturas.

Erdtman, 1952, relata que os grãos de pólen da família Caryophylaceae variam no tamanho, na forma, no tipo e número de aberturas e na ornamentação da exina. Markgraf e D'Antoni, 1978, encontraram para Arenarea andicola Gill. ex Hook. et Arn. um grão de pólen com características semelhantes em relação à forma, aberturas e ornamentação da exina, possuindo, porém, menor tamanho do que a espécie $C$. dicrotrichum.

COMMELINACEAE

NOME CIENTÍFICO: Tradescantia crassula Link et Otto

NOME VULGAR: Trapoeraba.

REFERÊNCIA: UPCB 14278.

ECOLOGIA: Planta de campo rupeste.

PÓLEN: prolato, com P/E = 1,65996. Tamanho médio. A exina é muito delgada. O pólen apresenta-se escabroso, com um colpo bem evidente.

Conforme Erdtman (1952), a família Commelinaceae não apresenta muitas variações (é estenopalinológica). Markgraf e D’Antoni (1978), em 
Commelina sp., encontraram dados que se enquadram nas descrições de Erdtman (1952).

Foram observadas, no Parque Estadual de Vila Velha, 3 espécies, sendo duas delas do gênero Tradescantia. Os dados obtidos na espécie em estudo está também de acordo com os dados relatados para a família por Erdtman (1952).

Tabela 1 - Dimensões dos diferentes eixos e vistas dos grãos de pólen das espécies analisadas (em $\mathrm{mm}$ ):

\begin{tabular}{|c|c|c|c|c|c|}
\hline Espécie & Vista & Eixo & Limites & $\bar{x} \pm \rho$ & $\sigma$ \\
\hline \multirow[t]{2}{*}{ Ditassa edmundoi } & \multirow[t]{2}{*}{ VPo } & $\mathrm{EM}$ & $55,5-68,0$ & $59,9 \pm 1,40$ & $\pm 4,44$ \\
\hline & & $\mathrm{Em}$ & $22,7-34,0$ & $26,6 \pm 1,12$ & $\pm 3,54$ \\
\hline \multirow[t]{3}{*}{ Vernonia nudiflora } & \multirow[t]{2}{*}{ VE } & EE & $30,0-33,7$ & $31,9 \pm 0,45$ & $\pm 1,41$ \\
\hline & & EP & $36,2-40,0$ & $38,1 \pm 0,46$ & $\pm 1,47$ \\
\hline & VP & EP & 33,1 - 36,2 & $34,4 \pm 0,23$ & $\pm 0,93$ \\
\hline \multirow[t]{3}{*}{ Begonia fruticosa } & \multirow[t]{2}{*}{ VE } & EE & $6,9-8,1$ & $7,62 \pm 0,12$ & $\pm 0,40$ \\
\hline & & EP & $11,2-11,9$ & $11,4 \pm 0,10$ & $\pm 0,30$ \\
\hline & VP & EP & $7,5-8,7$ & $7,9 \pm 0,16$ & $\pm 0,51$ \\
\hline \multirow[t]{2}{*}{ Berberis laurina } & \multirow[t]{2}{*}{ VU } & EM & 31,2 - 38,7 & $35,1 \pm 0,60$ & $\pm 1,90$ \\
\hline & & $\mathrm{Em}$ & $31,2-38,7$ & $35,5 \pm 0,62$ & $\pm 1,97$ \\
\hline \multirow[t]{3}{*}{ Jacaranda oxyphylla } & \multirow[t]{2}{*}{ VE } & EE & $30,0-42,5$ & $33,5 \pm 1,13$ & $\pm 3,57$ \\
\hline & & EP & $41,2-53,7$ & $48,6 \pm 1,38$ & $\pm 4,35$ \\
\hline & VP & EP & $26,9-31,2$ & $28,3 \pm 0,40$ & $\pm 1,25$ \\
\hline \multirow{3}{*}{$\begin{array}{l}\text { Cerastium dicrotrichum } \\
\text { Tradescantia crassula }\end{array}$} & VU & EU & $37,5-40,0$ & $38,6 \pm 0,31$ & $\pm 0,97$ \\
\hline & \multirow[t]{2}{*}{ VU } & $\mathrm{EM}$ & $31,2-35,0$ & $33,0 \pm 0,38$ & $\pm 1,21$ \\
\hline & & $\mathrm{Em}$ & $17,5-22,5$ & $19,9 \pm 0,54$ & $\pm 1,71$ \\
\hline
\end{tabular}

Legenda:

VPo: Vista da Políade

VE: Vista Equatorial

VP: Vista Polar

VU: Vista Única

EM: Eixo Maior

Em: Eixo Menor

EE: Eixo Equatorial

EP: Eixo Polar

EU: Eixo Único

$\overline{\mathrm{x}} \pm \rho$ : média aritmética \pm erro provável

$\sigma$ : desvio padrão 


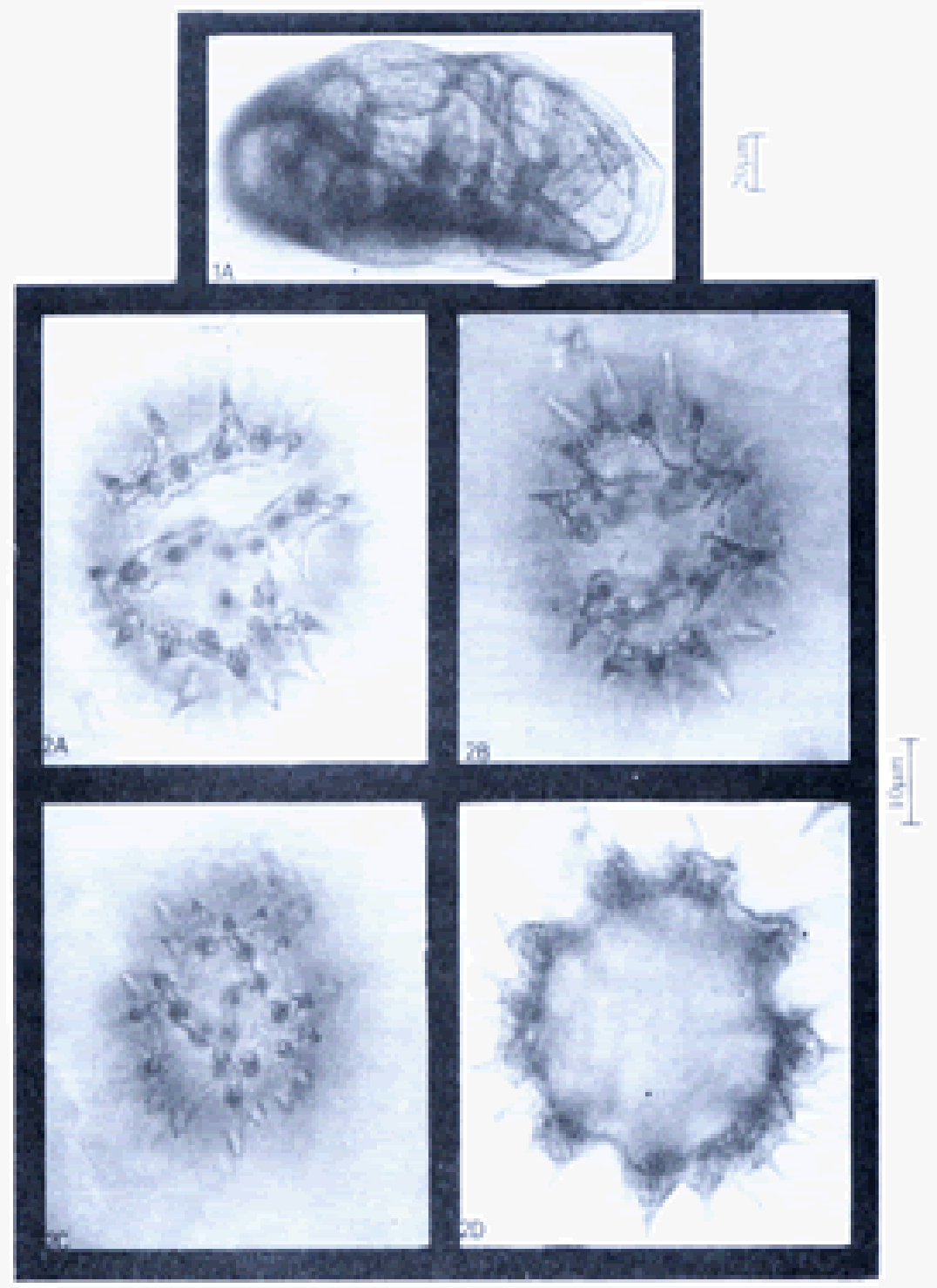

Prancha 1: Ditassa edmundoi: 1A, Políade; Vernonia nudiflora: 2A e 2B, PUBLICATIO UEPG - Biological and Health Sciences, 7 (1): 91-98, 2001. 
grão de pólen em Vista Equatorial, 2C e 2D, Vista Polar.

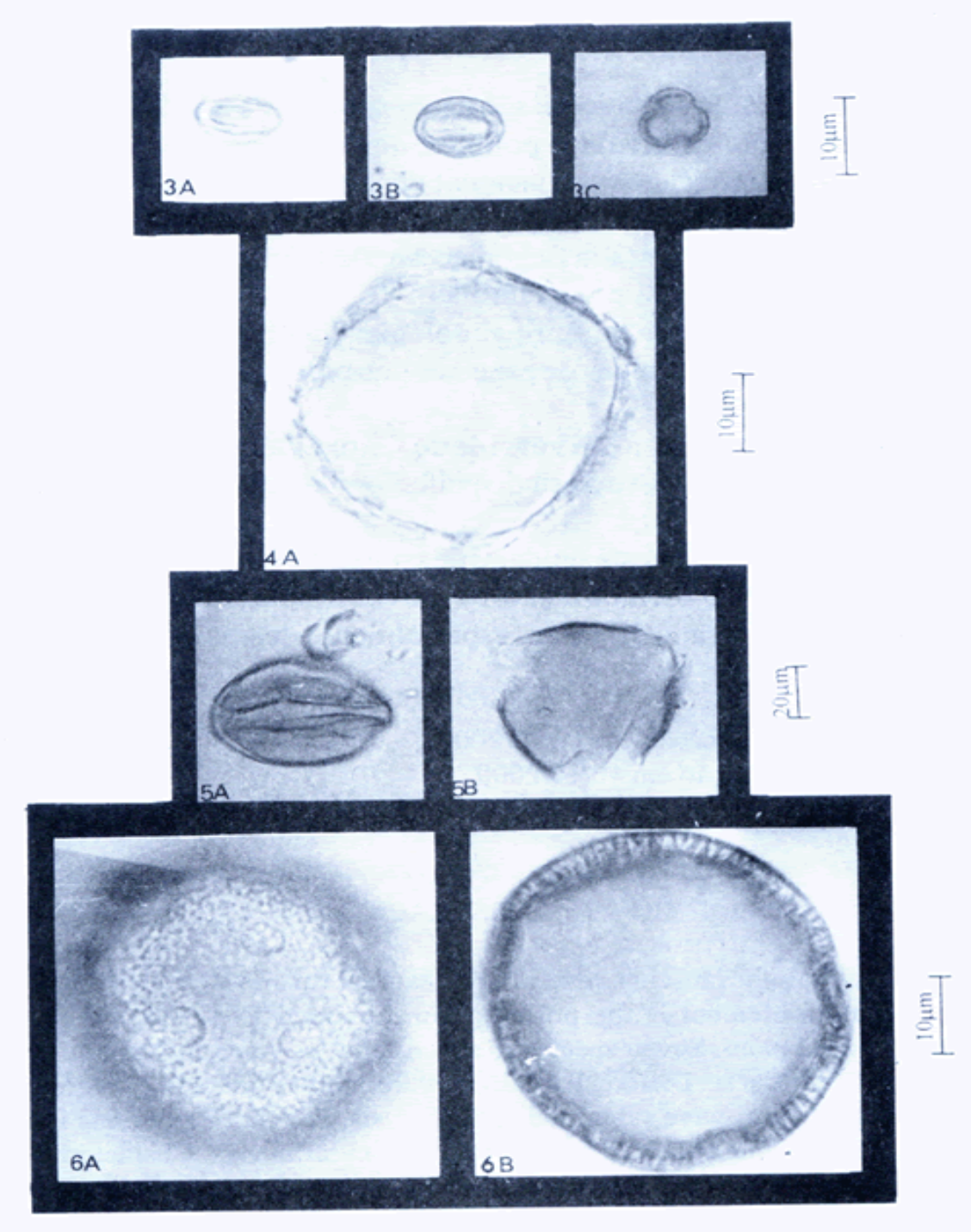

Prancha 2: Begonia fruticosa: 3A e 3B, grão de pólen em Vista Equatorial, 3C, Vista Polar; Berberis laurina: 4A, grão de pólen em Vista Única; Jacaranda oxyphylla: 5A, grão de pólen em Vista Equatorial, 5B, Vista Polar; Cerastium dicrotrichum: 6A e 6B, grão de pólen em Vista Única. 


\title{
4. Conclusões
}

Para a família Asteraceae são necessários mais estudos palinológicos, abrangendo maior diversidade polínica, devido ao grande número de espécies e representatividade populacional de muitas delas no Parque Estadual de Vila Velha.

Os taxa estudados para Begoniaceae, Berberidaceae e Commelinaceae são representativos para estas famílias.

Por Bignoniaceae ser euripalinológica, também se recomenda a análise da morfologia polínica das espécies dos outros dois gêneros ocorrentes na área de pesquisa.

Considerando a diversidade morfológica dos pólens de Caryophylaceae, indica-se o estudo palinológico de Polycarpaea corymbosa (L.) Lam.

Pela existência nesta unidade de conservação de espécies endêmicas e raras, sugere-se a elaboração de novos trabalhos de caracterização morfológica dos grãos de pólen, especialmente dessas espécies.

Recebido para publicação em 19/10/2000.

Aceito para publicação em 16/12/2000.

\begin{abstract}
Palynology presents several applications. In most of the time, the only identification element is the pollen grain, obtained from a determined and a countersigned plant. Seven species in seven families were analyzed, from UEPG and UPCB herbaria. Different families were studied planning future works in palynology. Grains were submitted to Acetolysis. For slides mounting it was used glycerol jelly, being followed by covering with paraffin wax. Characterization was made through several approaches, as: it forms, size and ornamentation of the exine. Analysed taxa were: Asclepiadaceae - Ditassa edmundoi Fontella et Valente, Asteraceae - Vernonia nudiflora Less, Begoniaceae - Begonia fruticosa (Kl.) A. DC., Berberidaceae - Berberis laurina Billb., Bignoniaceae - Jacaranda oxyphylla Cham., Caryophyllaceae - Cerastium dicrotrichum Fengl., Commelinaceae Tradescantia crassula Link et Otto. Asteraceae is markedly eurypalynous family.
\end{abstract}


Key words: pollen catalogue; Vila Velha State Park; Campos Gerais Region

\section{Agradecimentos}

Ao Departamento de Botânica da UFPR - Universidade Federal do Paraná pela utilização do fotomicroscópio e pela doação das amostras excicatadas retiradas do acervo do Herbário UPCB. À Pisa Florestal S.A., pela bolsa concedida à Bióloga Melissa Koch Fernandes de Souza. Ao CNPq/PIBIC/UEPG, pela bolsa de Iniciação Científica cedida para o então, acadêmico, Paulo Vitor Farago. Os agradecimentos também se estendem aos Departamentos de Biologia Geral e de Química da UEPG - Universidade Estadual de Ponta Grossa, pelo empréstimo de reagentes químicos e equipamentos, fundamentais ao desenvolvimento do trabalho.

Endereço para contato: sibele_takeda@uol.com.br

\section{REFERÊNCIAS}

1 CERVI, A. C.; HATSCHBACH, G. Flora. In: ROCHA, C. H.; MICHALIZEN, V.; PONTES FILHO, A. (Coords.) Plano de Integração Parque Estadual de Vila Velha - Rio São Jorge. Ponta Grossa: Ituphava S/C e Prefeitura Municipal de Ponta Grossa, 1990.

2 ERDTMAN, G. Pollen morphology and plant taxonomy - Angiosperm. Waltham: Chonica Botanica C.O, 1952. 540 p.

3 ERDTMAN, G. Handbook of Palynology - Morphology - Taxonomy - Ecology. New York: Hafner Publishing C.O., 1969. 485 p.

4 HATSCHBACH, G.; MOREIRA FILHO, H. Catálogo florístico do Parque Estadual de Vila Velha (Estado do Paraná, Brasil). Boletim da Universidade Federal do Paraná - Botânica, n. 28, set. 1972.

5 MAACK, R. Geografia física do Paraná. 2. ed. Curitiba: Liv. José Olympio, 1968. $442 \mathrm{p}$.

6 MARKGRAF, V.; D’ANTONI, H. L. Pollen Flora of Argentina. Tucson: The 
University of Arizona Press, 1978. 208 p.

7 MIRANDA, M. M. B.; ANDRADE, T. A. P. Fundamentos de Palinologia. Fortaleza: Imprensa Universitária da Universidade Federal do Ceará, 1990. 99 p.

8 PARANÁ. Secretaria de Estado de Meio Ambiente. Lista vermelha de plantas ameaçadas de extinção no Estado do Paraná. Curitiba: SEMA/GTZ, 1995.

9 REIS, I. J. M. Estudo da morfologia dos grãos de pólens dos gêneros da tribo Eupatorieae no Rio Grande do Sul. 1979. 116 p. Dissertação (Mestrado em Ciências Biológicas) - Departamento de Botânica, Universidade Federal do Rio Grande do Sul, Porto Alegre.

10 SAlgado-LABOURIAU, M. L. Palinologia dos Cerrados. An. Acad. Brasil. Cienc. São Paulo, 38 (supl.): 187-206. 1966.

11 SALGADO-LABOURIAU, M. L. Contribuição à Palinologia dos Cerrados. São Paulo: Acad. Brasil. Ciênc., 1973. 291 p.

12 STIX, E. Pollenmorphologische Untersuchengen an Compositem. Grana Palynol. Müchen, 2 (2): 41-126. 1960.

13 TOMB, S. A.; LARSON, D. A.; SKVARLA, J. J. Pollen morphology and detailed structure of family Compositae. Tribe Cichorieae. I - Subtribe Stephanomeriineae. Am. J. Bot., 61 (5): 486-498. 1974. 